\title{
Cold Potassium Needs No Ovens
}

\author{
A technique for quickly trapping ultracool molecules can now work on \\ alkali metal atoms.
}

By Rahul Rao

C

urrently, researchers wishing to trap and tinker with ultracold atoms mainly rely on ovens, which produce gaseous atoms at their vapor pressure, as atomic sources. The high temperatures and low fluxes of atoms from such sources mean that cooling and loading the atoms into traps can be slow, especially for atoms with high boiling points. Now, Zack Lasner at the Harvard-MIT Center for Ultracold Atoms and colleagues have sped things along by instead using a cryogenic buffer gas beam source (CBGB), which can load a trap with atoms at a rate independent of their boiling point [1].

Until now, CBGBs have mainly been used for molecular physics, although they have also been shown to work with lanthanide atoms. As alkali metals are still the go-to atoms for cold-atom physics, expanding the use of CBGBs to other atomic species is important. With this goal, Lasner and colleagues use a standard CBGB as a source of potassium atoms.

The process begins with a cryogenic cell in which a buffer gas (helium) is held at about $6 \mathrm{~K}$. A laser is fired into the cell at a potassium-chloride target. Potassium atoms released from the

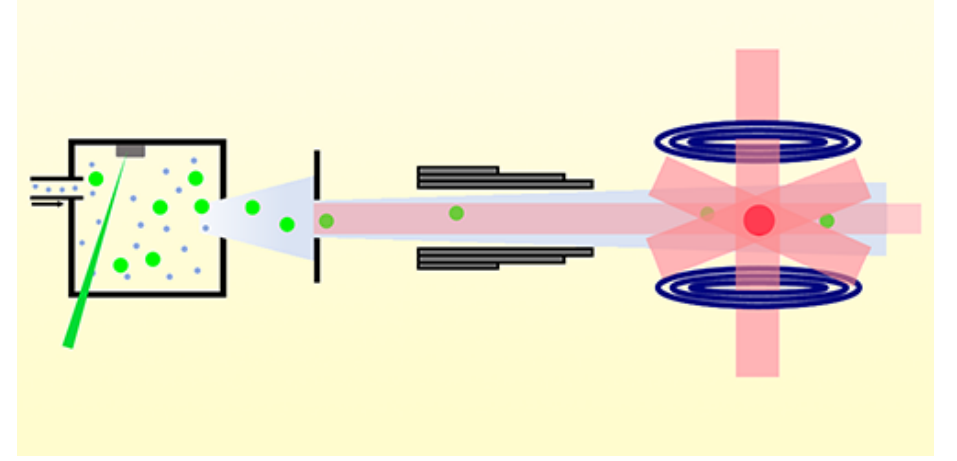

target are cooled by the helium, then file out of the cell and through a Zeeman slower, which decelerates them as they enter the trap.

The researchers show that a trap loaded from their CBGB source can achieve atom yields and densities comparable to state-of-the-art oven-based traps, but taking just $10 \mathrm{~ms}$ instead of the 1-20 s needed for a conventional oven source. Beyond alkali atoms, the researchers say that the method could be useful in trapping transition-metal atoms, which are tricky to handle with traditional oven-based methods.

Rahul Rao is a freelance science writer based in New York.

\section{REFERENCES}

1. Z. Lasner et al., "Fast and high-yield loading of a $\mathrm{D}_{2}$ magneto-optical trap of potassium from a cryogenic buffer-gas beam," Phys. Rev. A 104, 063305 (2021).

Credit: Z. Lasner et al. [1] 